\title{
Coloration of Polypropylene Gel and Temperature Conditions for Gel Formation
}

\author{
Hitoshi Fujimatsu, ${ }^{\dagger}$ Yukinori Ideta, Hitoshi NAKamura, Hisanao Usami, \\ and Shinji OGASAWARA

\begin{abstract}
Department of Fine Materials Engineering, Faculty of Textile Science and Technology,
\end{abstract} \\ Shinshu University, 3-15-1 Tokida, Ueda, Nagano 386-8567, Japan
}

(Received August 7, 2000; Accepted May 25, 2001)

\begin{abstract}
We investigated, on the basis of light-scattering theory, the relationship between the color of polypropylene gel and the refractive index in the vicinity of the interface of polypropylene networks in the gel when prepared at different cooling temperatures after dissolving the polypropylene at $160^{\circ} \mathrm{C}$. The color of the gels was found to differ depending on the cooling temperature upon gel formation. SEM images of these gels showed polypropylene networks with smaller diameters with decreases in the cooling temperature. Based on measurements using the maximum transmittance method, it was found that the origin of color changes in the gels is a shift in the wavelength dispersion of the refractive index for polypropylene to a higher refractive index. Changes in the solvent content of polypropylene in the gels, which were evaluated using experimental values of the refractive indices and densities for the solvent and polypropylene, revealed that the solvent causes swelling of the polypropylene interface which is a source of light- scattering. The degree of swelling appears to depend upon the cooling temperature when gels are prepared by cooling beyond the glass transition temperature of polypropylene.

KEY WORDS Coloration / Thermochromism / Polypropylene / Xylene / Surface / Wavelength Dispersion of Refractive Index /
\end{abstract}

We have previously reported that polymer gels in organic solvents such as polypropylene and poly-1butene develop colors, although polypropylene and poly-1-butene themselves are colorless, with the colors changing with increases in temperature. This coloring phenomenon has been considered to be due to selective light-scattering, as the transmitted-light spectra of the gels forms a rough reflected image of the scatteredlight spectra, which are measured in the direction perpendicular to the incident light. ${ }^{1,2}$ We have more recently demonstrated that the development of coloration in polypropylene gels can be explained due to selective light-scattering on the basis of differences in the lightdispersive power between solvent and polypropylene, and to size-controlled polypropylene networks formed in the gel. ${ }^{3-11}$ Furthermore, it has been experimentally proved that the thermochromic phenomenon in the gel can be explained consistently using the same logic. However, the differences in color between gels prepared at different cooling temperatures have not been mentioned.

In the present paper, we provide details regarding the relationship between gel color and the refractive index in the vicinity of the interface of polypropylene networks gels prepared under confined conditions.

${ }^{\dagger}$ To whom correspondence should be addressed.

\section{EXPERIMENTAL}

\section{Materials}

The polypropylene used in the present study was narrowly dispersed isotactic polypropylene with an average molecular weight of approximately $20.0 \times 10^{4}$, which was supplied by the Research and Institute Center of Chisso Petrochemical Co. $O$-Xylene was used as a solvent for gelling of polypropylene.

Polypropylene gel was prepared in glass tubes as follows: $0.2 \mathrm{~g}$ of polypropylene and $10 \mathrm{~mL}$ of solvent were placed in a glass tube, which was sealed in a flame. After the polypropylene was dissolved completely at $160^{\circ} \mathrm{C}$, the polypropylene solution was cooled at given temperatures in the range of $-195^{\circ} \mathrm{C}$ to $50^{\circ} \mathrm{C}$, which resulted in gel formation.

\section{Methods}

The wavelength-dependent transparency was measured using a Shimadzu MPS-2000 spectrophotometer. The temperature was controlled by circulating water through the cell jacket. The light-scattered spectrum of the gel in the direction perpendicular to the incident light was measured using a Shimadzu RF-5000 fluorescent spectrophotometer with a temperature controller. The obtained spectra were incorporated into the memory of a computer to be corrected by the spectrum of a Xenon-lamp, which was the light source for the spec- 
trophotometer.

The refractive index of organic solvents was measured at a wavelength of 450 to $700 \mathrm{~nm}$ at a constant room temperature of $25^{\circ} \mathrm{C}$ by an Atago Abbe-type refractometer Type-1T equipped with a spectroscopic monochrometer.

Electron micrographs of gels were obtained using a Akashi type ALPHA 30A scanning electron microscope. The specimens were prepared by depositing gold under vacuum below $10^{-5}$ Torr after the polypropylene gels were freeze-dried.

The density of polypropylene in the gel was estimated from the density of the gel measured at $25^{\circ} \mathrm{C}$. The density of the gel was measured using a Shibayama SS-D-200 glass tube vibrator type density meter.

\section{RESULTS AND DISCUSSION}

\section{Spectra of Polypropylene Gels and Conditions for Preparing Gels}

We have previously reported that only polypropylene gels formed by rapid cooling have clear coloration. ${ }^{1}$ This phenomenon is considered to be of importance in connection with the coloration mechanism. Figure 1 shows the transmittance spectra of $2 \mathrm{~g} / \mathrm{v}-\%$ of polypropylene gels in $o$-xylene after reaching equilibrium at $25^{\circ} \mathrm{C}$ that had been prepared at various temperatures after being dissolved in the solvent at $160^{\circ} \mathrm{C}$.

As can be seen from Figure 1, the polypropylene gel prepared at higher temperature resulted in a shift in the transmittance peak to a shorter wavelength. While the gels prepared by cooling at temperatures below $-10^{\circ} \mathrm{C}$ showed somewhat similar spectra, the shape of the spectra prepared by cooling beyond $20^{\circ} \mathrm{C}$ was changed. In particular, the spectrum of the gel cooled at $40^{\circ} \mathrm{C}$ has a transmittance peak at approximately $400 \mathrm{~nm}$ and

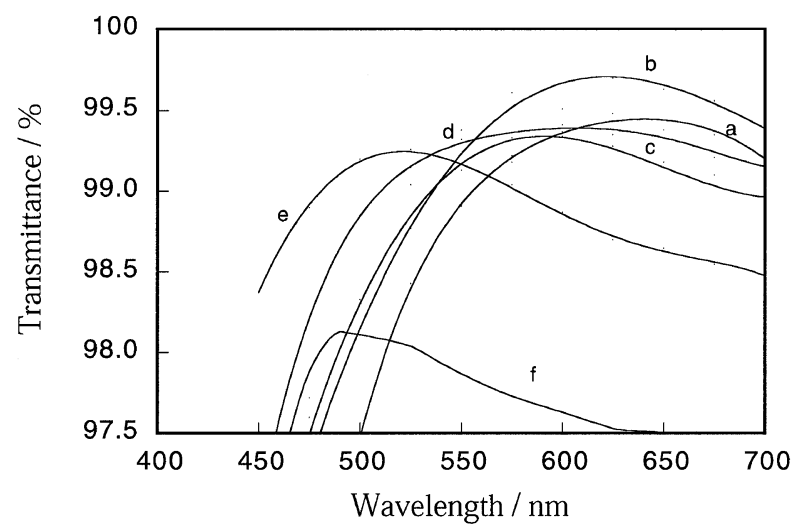

Figure 1. Transmittance spectra for $2 \mathrm{~g} / \mathrm{v}-\%$ polypropylene gels in $o$-xylene after reaching equilibrium at $30^{\circ} \mathrm{C}$, that were prepared at different cooling temperatures after polypropylene was dissolved in the solvent at $160^{\circ} \mathrm{C}$ : (a) $-195^{\circ} \mathrm{C}$, (b) $-40^{\circ} \mathrm{C}$, (c) $-20^{\circ} \mathrm{C}$, (d) $0^{\circ} \mathrm{C}$, (e) $20^{\circ} \mathrm{C}$, (f) $40^{\circ} \mathrm{C}$.
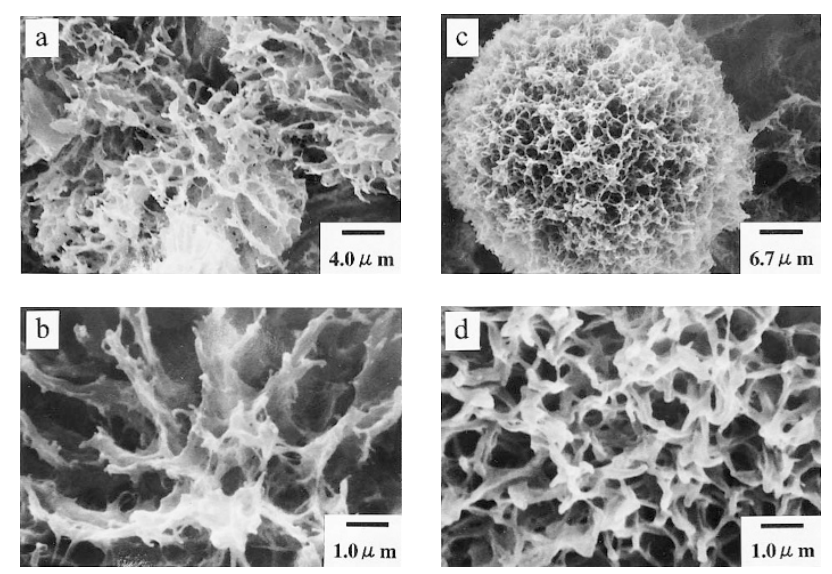

Figure 2. SEM images for $2 \mathrm{~g} / \mathrm{v}-\%$ polypropylene gel in $o$-xylene prepared at different cooling temperatures. $-50^{\circ} \mathrm{C}$ : magnification (a) $\times 2500$, (b) $\times 10000$ ), and $50^{\circ} \mathrm{C}$ : magnification (c) $\times 1500$, (d) $\times 10000$, with scale shown.

lower transmittance over the whole visible region. The gel appears semi-transparent to the naked eye. Why do such a change in spectrum occur? First, the change in the crystal system of polypropylene in the gel was examined by X-Ray diffraction, no useful evidence was obtained. Subsequently, to examine the microstructure of these gels, SEM observations were carried out. The specimen was prepared by depositing gold onto freezedried gel.

To illustrate the experimental results, SEM images of $2 \mathrm{~g} / \mathrm{v}-\%$ polypropylene gels prepared by cooling at $-50^{\circ} \mathrm{C}$ and $50^{\circ} \mathrm{C}$ are shown in Figure 2. For the gel prepared by rapid cooling at $-50^{\circ} \mathrm{C}$, spongy aggregates approximately $10 \mu \mathrm{m}$ in diameter are observed. The high-magnification SEM image reveals a network with a diameter of approximately 500 to $1000 \mathrm{~nm}$, corresponding to wavelengths in the visible region. In contrast, for the gel prepared by gradual cooling at $50^{\circ} \mathrm{C}$, spherulites approximately $50 \mu \mathrm{m}$ in diameter are observed. The high-magnification SEM image shows that the gel is composed of a polypropylene network of higher density than that of the gel at $-50^{\circ} \mathrm{C}$. Although these SEM images do not necessarily show the polypropylene networks themselves in the gel states, as observed for freeze-dried gels, relative differences between polypropylene networks in the gel states should be reflected in the SEM images. These SEM observations suggest that gels prepared at lower cooling temperatures have a structural micron order favorable to scattering visible light. But the spectrum change of the gels prepared at different cooling temperatures is not explicable from these results. Actually, in the gel state, the solvent must be incorporated into the polypropylene networks observed in the SEM. 


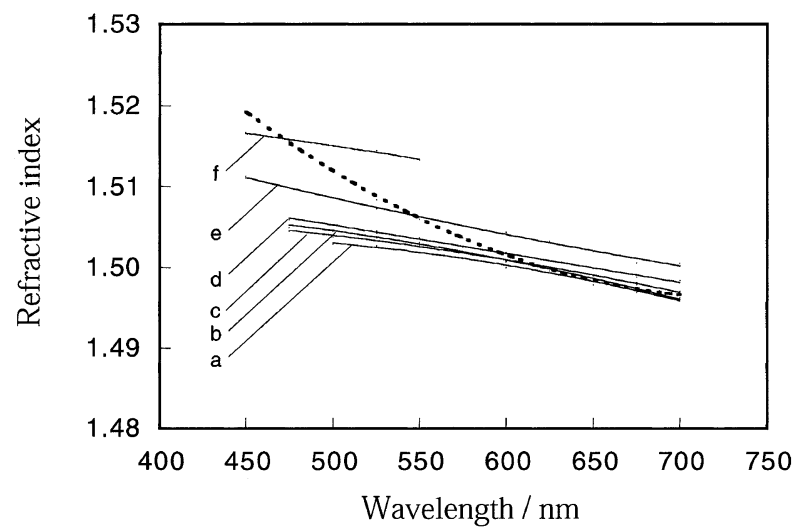

Figure 3. Wavelength dispersions of refractive index for polypropylene (solid line) in gels prepared at different cooling temperatures and for $o$-xylene (dotted line) at $25^{\circ} \mathrm{C}$ : (a) $-195^{\circ} \mathrm{C}$, (b) $-40^{\circ} \mathrm{C},(\mathrm{c})-20^{\circ} \mathrm{C},(\mathrm{d}) 0^{\circ} \mathrm{C}$, (e) $20^{\circ} \mathrm{C}$, (f) $40^{\circ} \mathrm{C}$.

Refractive Index Dispersions of Polypropylene in the Gels Prepared at Different Cooling Temperatures

We have recently demonstrated that the development of coloration in polypropylene gels can be explained by selective light-scattering with this conclusion being based on differences in the light-dispersive power of solvents and polypropylene, and the size-controlled polypropylene networks formed in the gels. According to the light-scattering theory, the shift of the peak in the transmittance spectra showed in Figure 1 may reflect the change in the relative refractive index between polypropylene and the solvent. As the measurements of the spectra were carried out at $25^{\circ} \mathrm{C}$, the refractive index of the solvent must have remains constant. Accordingly, the possibility of a change in the refractive index of polypropylene in the gels is present. If it is found that the refractive index of polypropylene in the gels changes depending on the cooling temperature during gel preparation, the validity of this hypothesis may be confirmed. Therefore, the wavelength dispersion of the refractive index of polypropylene in the gel was estimated by a similar method to that used previously. The results are showed in Figure 3, accompanying wavelength dispersions of the refractive index of the $o$-xylene used as a solvent. Refractive index dispersions of polypropylene in gels have been found to shift upward with increases in the cooling temperature during gel formation. ${ }^{12}$ Therefore, it is apparent that the point at which dispersion curves for polypropylene cross those of the solvent shift to a shorter wavelength increases in the cooling temperature. This result corresponds to the experimentally observed color changes from blue to cloudy white through reddish violet.

As can be seen from Figure 3, dispersion curves in cooling temperatures below $0^{\circ} \mathrm{C}$ show a very small change, but those in cooling temperatures beyond $20^{\circ} \mathrm{C}$

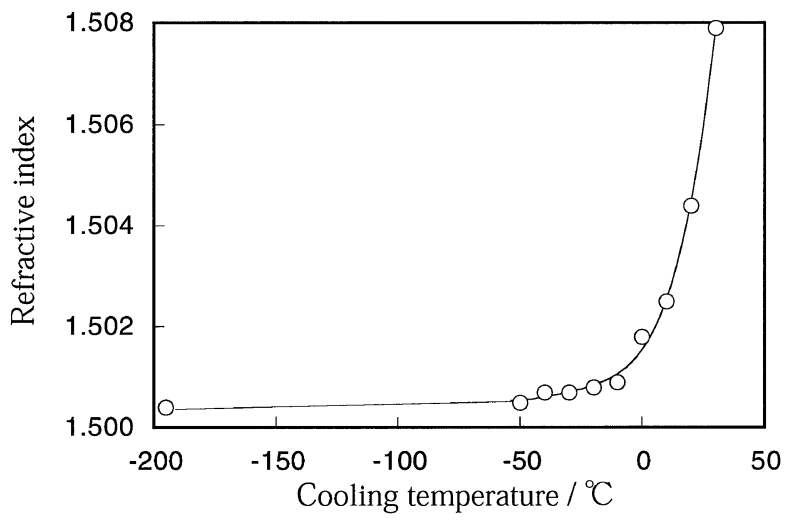

Figure 4. Relationship between the refractive index of polypropylene in gels at $600 \mathrm{~nm}$ and the cooling temperature for gel formation. The refractive indices of polypropylene in gels are estimated by the maximum-transmittance method.

appear to change rapidly. In Figure 4, we have plotted the refractive index of polypropylene at a wavelength of $600 \mathrm{~nm}$, shown in Figure 3, against cooling temperature. It is apparent that the refractive index of polypropylene in the gel begins to increase with temperature in the vicinity of $-20^{\circ} \mathrm{C}$, which is likely due to the differences in the crystallization of polypropylene dependent on the different cooling temperatures. The glass transition point $T_{\mathrm{g}}$ of polypropylene employed in this study was measured by the DSC method, and was found to be $-21^{\circ} \mathrm{C}$. The obtained $T_{\mathrm{g}}$ is in fair agreement with the change point of the refractive index shown in Figure 4. In general, it is well known that polymers behave like solids below $T_{\mathrm{g}}$, while beyond $T_{\mathrm{g}}$ they behave like rubber. When the polypropylene solution dissolved at high temperature was cooled at temperatures below $T_{\mathrm{g}}$, it became difficult for the microcrystals of polypropylene formed in the gel to grow. In contrast, beyond $T_{\mathrm{g}}$ the microcrystals could grow dependent on the cooling temperature. These difference can be regarded as reflecting the change in the refractive index.

\section{Density of Polypropylene in Gel}

In general, it is known that the refractive index of a substance is affected by density of the substance. The relationship between the refractive index and the density of a substance has been derived according to the Clausius-Mosotti equation as follows:

$$
\left(n^{2}-1\right) /\left(n^{2}+2\right)=N \alpha_{\mathrm{a}} / 3 \varepsilon_{0}
$$

where $n$ is the refractive index, $N$ is the density per molecule, $\alpha_{\mathrm{a}}$ is the polarizability per molecule, and $\varepsilon_{0}$ is the dielectric constant under vacuum. The $\left(n^{2}-\right.$ $1) /\left(n^{2}+2\right)$ term is directly proportional to $N$. Accordingly, the polypropylene density in the gel was estimated from the gel density measured at $25^{\circ} \mathrm{C}$ using a glass tube vibrator type density meter. In Fig- 


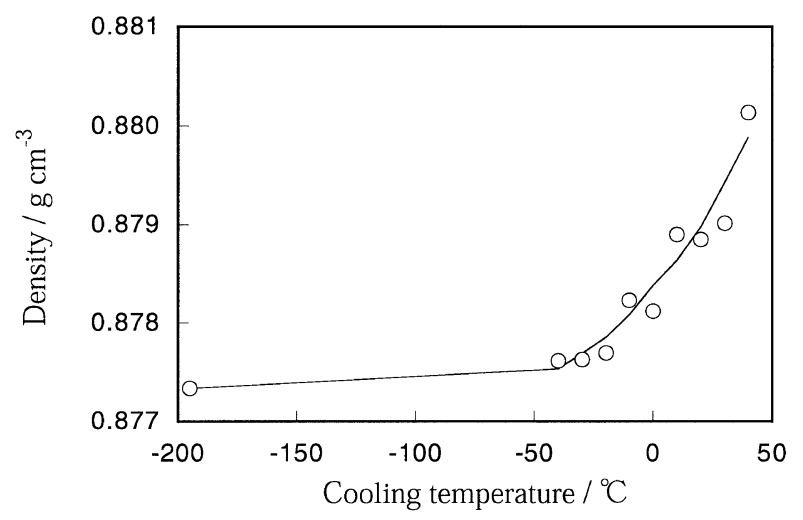

Figure 5. Relationship between the density of polypropylene in the gels and the cooling temperature. The densities of polypropylene in the gels are estimated from the densities of the gels measured at $25^{\circ} \mathrm{C}$ using glass tube vibrator type density meter.

ure 5, obtained values are plotted against the cooling temperature for gel formation. The change in density is analogous to that of the refractive index. All of these values are intermediate between the density of the polypropylene sample $0.882 \mathrm{~g} \mathrm{~cm}^{-3}$ and that of $o$-xylene, $0.869 \mathrm{~g} \mathrm{~cm}^{-3}$, suggesting that the measured density values are average values of the density of the solvent and polypropylene, and hence the interface of the polypropylene network, which is a source of lightscattering, swells due to contact with the solvent.

\section{Solvent Content of Polypropylene in the Gel}

It is of interest in connection with the light-scattering ability to know the solvent content of polypropylene in the gel. It is known that the relationship between the refractive index and composition in a mixture is derived by the extended Clausius-Mosotti's equation, as follows.

$$
\begin{aligned}
\left(n^{2}-1\right) /\left(n^{2}+2\right) & =(1-c)\left(\rho / \rho_{1}\right)\left\{\left(n_{1}^{2}-1\right) /\left(n_{1}^{2}+2\right)\right\} \\
& +c\left(\rho / \rho_{2}\right)\left\{\left(n_{2}^{2}-1\right) /\left(n_{2}^{2}+2\right)\right\}
\end{aligned}
$$

where $c$ is the weight fraction of component $2, n$ is the refractive index of the mixture, $n_{1}$ is the refractive index of component $1, n_{2}$ is the refractive index of component $2, \rho$ is the density of the mixture, $\rho_{1}$ is the density of component 1 , and $\rho_{2}$ is the density of component 2 .

The solvent content of polypropylene in the gel was evaluated using the experimental values of refractive indices and densities for the solvent and polypropylene according to the above equation. In Figure 6, the calculated solvent contents were plotted against the cooling temperature for gel formation. The result corresponds relatively well to that shown in Figure 5. It can be seen that the solvent content begins to decrease rapidly beyond $-20^{\circ} \mathrm{C}$. Taking into account the results shown in Figure 4, the solvent content is thought to decrease with a concomitant increase in the crystallization

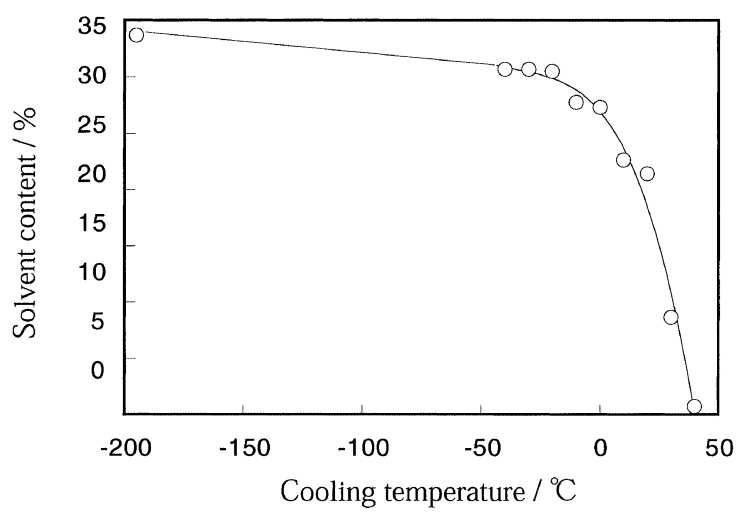

Figure 6. Relationship between the solvent content of polypropylene in the gels and the cooling temperature.

of polypropylene in the gel. As these results were obtained based on experimental values regarding the refractive index of polypropylene in the gel, they are considered to reflect the interface state of polypropylene in the gel. Accordingly, we conclude that changes in this interface cause changes in the refractive index dispersion of polypropylene in the gels, as shown in Figure 3, as well as the different gel colors, even when observed at a fixed temperature, that correspond to differences in the cooling temperature during gel formation.

Acknowledgment. The present work was partly supported by Grant-in-Aid for COE Research (10CE2003) from the Ministry of Education, Science, Sports and Culture of Japan. The authors wish to express many thanks to Prof. S. Hayashi and Prof. T. Hirai of Shinshu University for helpful suggestions and discussions, and Mr. I. Namiki of Technical Center, Chisso Petrochemical Industry Co. for supplying the polypropylene samples.

\section{REFERENCES}

1. H. Fujimatsu and S. Kuroiwa, Colloid Polym. Sci., 265, 938 (1987).

2. H. Fujimatsu, S. Ogasawara, H. Ihara, T. Takashima, K. Toyaba, and S. Kuroiwa, Colloid Polym. Sci., 266, 688 (1988).

3. H. Fujimatsu, Y. Ideta, H. Nakamura, H. Usami, and S. Ogasawara, Polym. J., 33, 89 (2001).

4. G. Mie, Ann. Phys. (Orland, FL), 25, 377 (1908).

5. L. Rayleight, Proc. R. Soc. London, Ser. A, 84, 25 (1911).

6. R. Gans, Ann. Phys. (Orland, FL), 76, 29 (1925).

7. P. Debye and R. Mecke, Forts. Roentgenforschung, 2, 1 (1931).

8. P. Debye, J. Phys. Colloid. Chem., 51, 18 (1947).

9. Vande Hulst, "Light Scattering by small particles", John Willy and Sons, Inc., New York, N.Y., 1957.

10. W. Heller, J. Chem. Phys., 14, 9 (1946).

11. M. Nakagaki and W. Heller, J. Appl. Phys., 27, 975 (1956).

12. S. Hayashi, J. Xu, K. Asada, and T. Hirai, J. Colloid Interface Sci., 163, 315 (1994). 